\title{
On the ergodic and spectral properties of generalized Boole transformations. I
}

\author{
Anatoly K. Prykarpatsky and Jacek Feldman
}




\title{
ON THE ERGODIC AND SPECTRAL PROPERTIES OF GENERALIZED BOOLE TRANSFORMATIONS. I
}

\author{
ANATOLIY K. PRYKARPATSKY AND JACEK FELDMAN
}

[Received: May 23, 2005]

\begin{abstract}
The invariant ergodic measures for generalized Boole type transformatins are studied making use of the invariant quasi-measure approach, based on some special solutions to the Frobenius-Perron operator.
\end{abstract}

Mathematics Subject Classification: Primary 34A30, 34B05 Secondary 34B15

Keywords: generalized Boole transformations, ergodic dynamical systems, invariant quasi-measures, Frobenius-Perron operator

\section{InTROduction}

We will consider the following generalized Boole transformation

$$
\mathbb{R} \ni y \rightarrow \varphi(y):=\alpha y+a-\sum_{j=1}^{N} \frac{\beta_{j}}{y-b_{j}} \in \mathbb{R},
$$

where $a$ and $b_{j} \in \mathbb{R}, j=\overline{1, N}$, are some real and $\alpha, \beta_{j} \in \mathbb{R}_{+}, j=\overline{1, N}$, are positive parameters. It generalizes that classical Boole transformation [1] $\mathbb{R} \ni y \mapsto \varphi(y):=$ $y-1 / y \in \mathbb{R}$, which appeared to be ergodic [2] with respect to the invariant standard infinite Lebesgue measure on $\mathbb{R}$. In the case where $\alpha=1, a=0$, the similar ergodicity result was proved in [3-5] making use of the specially despised inner function notion. The related spectral properties were in part studied in [5]. In spite of these results the case $\alpha \neq 1$ still persists to be challenging as the only related result [6] concerns the following special case of (1.1): $\mathbb{R} \ni y \mapsto \varphi(y):=\alpha y-\beta / y \in \mathbb{R}$ for $0<\alpha<1$ and arbitrary $\beta \in \mathbb{R}_{+}$. The corresponding invariant measure appeared to be finite absolutely continuous with respect to the Lebesgue measure on $\mathbb{R}$ and equal to

$$
d \mu(x):=\frac{\sqrt{\beta(1-\alpha)} d x}{\pi\left[x^{2}(1-\alpha)+\beta\right]},
$$

The first author was supported in part by a local AGH grant. 
where $x \in \mathbb{R}$. The ergodicity for the invariant measure (1.2) now can be easily proved. Recall here, concerning a general nonsingular mapping $\varphi: \mathbb{R} \rightarrow \mathbb{R}$, the problem of constructing the measure preserving ergodic measures is analized [6,7] by means of studying the spectral properties of the adjoint Frobenius-Perron operator $\hat{T}_{\varphi} \varrho$ : $L_{2}(\mathbb{R} ; \mathbb{R}) \rightarrow L_{2}(\mathbb{R} ; \mathbb{R})$, where, by definition,

$$
\hat{T}_{\varphi} \varrho(x):=\sum_{y \in\left\{\bar{\varphi}^{\prime}(x)\right\}} \varphi(y) J_{\varphi}^{-1}(y)
$$

for any $\varrho \in L_{2}\left(\mathbb{R} ; \mathbb{R}_{+}\right)$and $J_{\varphi}^{-1}(y):=\left|\frac{d \varphi(y)}{d y}\right|, y \in \mathbb{R}$. Then if $\hat{T}_{\varphi} \varrho=\varrho, \varrho \in L_{2}\left(\mathbb{R} ; \mathbb{R}_{+}\right)$, then the expression $d \mu(x):=\varrho(x) d x, x \in \mathbb{R}$, will be an invariant, in general infinite, measure with respect to the mapping $\varphi: \mathbb{R} \rightarrow \mathbb{R}$.

Another way to finding a general algorithm for finding such an invariant measure was devised in $[8,9]$, making use of the generating measure function method.

Below we will study another special cases of the generalized Boole transformation (1.1), for which we deliver the corresponding invariant measures and prove the related ergodicity and spectral properties.

\section{INVARIANT MEASURES AND ERGODIC TRANSFORMATIONS}

We will start by analyzing the following Boole type surjective transformation

$$
\mathbb{R} \ni y \rightarrow \varphi(y):=\alpha y+a-\frac{\beta}{y-b} \in \mathbb{R}
$$

for any $a, b \in \mathbb{R}$ and $2 \beta:=\gamma^{2} \in \mathbb{R}_{+}$. The transformation (2.1) at $\alpha=1 / 2$ and $b=2 a \in \mathbb{R}$ will be measure preserving with respect to a measure like (1.2). Namely, the following lemma holds.

Lemma 2.1. The Boole type mapping (2.1) is measure preserving with respect to the measure

where $x \in \mathbb{R}$ and $\gamma^{2}:=2 \beta \in \mathbb{R}_{+}$.

$$
d \mu(x):=\frac{|\gamma| d x}{\pi\left[(x-2 a)^{2}+\gamma^{2}\right]},
$$

Proof. A proof follows easily from the fact that the function

$$
\varrho(x):=\frac{\gamma}{\pi\left[(x-2 a)^{2}+\gamma^{2}\right]}
$$

satisfies for all $x \in \mathbb{R} \backslash\{2 a\}$ the determining condition (1.3):

$$
\hat{T}_{\varphi} \varrho(x):=\sum_{I} \varrho\left(y_{ \pm}\right) y_{ \pm}^{\prime}(x)
$$

where, by definition, $\varphi\left(y_{ \pm}(x)\right):=x$ for any $x \in \mathbb{R}$. The relation (2.4) is, evidently, equivalent to the next invariance condition

$$
\sum_{ \pm} d \mu\left(y_{ \pm}(x)\right)=d \mu(x):=\mu(d x)
$$


for any infinitesimal subset $d x \subset \mathbb{R}$.

The question about the ergodicity of mapping (2.1) is solved here easily by the following theorem.

Theorem 2.2. Measure (2.3) is ergodic with respect to transformation (2.1) at $\alpha=1 / 2$ and $b=2 a \in \mathbb{R}$ as such one is equivalent to the canonical ergodic mapping $\mathbb{R} / \pi \mathbb{Z} \ni s \mapsto \psi(s):=2 s \bmod (\pi Z) \in \mathbb{R} / \pi \mathbb{Z}$ with respect to the standard Lebesgue measure on $\mathbb{R} / \pi \mathbb{Z}$.

Proof. Put by definition $\mathbb{R} / \pi \mathbb{Z} \ni s \mapsto \xi(s)=y \in \mathbb{R}$, where

$$
\xi(s):=\gamma \operatorname{ctg} s+2 a,
$$

Then transformation (2.1) at $\alpha=1 / 2, b=2 a \in \mathbb{R}$ and $\gamma^{2}:=2 \beta \in \mathbb{R}_{+}$yields under mapping (2.6)

$$
\begin{aligned}
\varphi(y) & =\varphi(\xi(s))=\frac{\gamma}{2} \operatorname{ctg} s+2 a-\frac{\gamma}{2} \operatorname{tg} s=\frac{\gamma\left(\cos ^{2} s-\sin ^{2} s\right)}{2 \sin s \cos s}+2 a \\
& =\gamma \frac{\cos 2 s}{\sin 2 s}+2 a=\gamma \operatorname{tg} 2 s+2 a:=\xi(2 s)
\end{aligned}
$$

for any $s \in \mathbb{R} / \pi \mathbb{Z}$. Relation (2.7) means that transformation (2.1) is conjugated [5,7] with the transformation

$$
\mathbb{R} / \pi \mathbb{Z} \ni s \longmapsto \psi(s)=2 s \bmod (\pi \mathbb{Z}) \in \mathbb{R} / \pi \mathbb{Z},
$$

that is the following diagram is commutative:

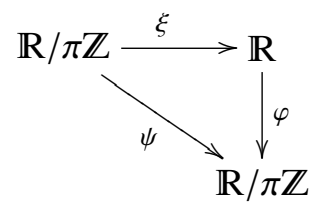

It is easy now to check that the measure (2.2) under the conjugation (2.9) transforms into the standard normalized Lebesgue measure on $\mathbb{R} / \pi \mathbb{Z}$ :

$$
\left.d \mu(x)\right|_{x=\gamma \operatorname{ctg} s+2 a}=\frac{d s \gamma^{2}|d(\operatorname{ctg} s) / d s|}{\pi\left(\gamma^{2} \operatorname{ctg}^{2} s+\gamma^{2}\right)}=\frac{1}{\pi} \frac{\sin ^{2} s \cdot \sin ^{-2} s d s}{\cos ^{2} s+\sin ^{2} s}=\pi^{-1} d s,
$$

where $s \in \mathbb{R} / \pi \mathbb{Z}$. The infinitesimal measure $\pi^{-1} d s$ on $\mathbb{R} / \pi \mathbb{Z} \ni s$ as well as the infinitesimal measure (2.2) on $\mathbb{R}$ are normalized, being thus probabilistic. Now it is enough to make use of the fact that the measure $\pi^{-1} d s$ on $\mathbb{R} / \pi \mathbb{Z} \ni s$ on the interval $[0, \pi] \simeq \mathbb{R} / \pi \mathbb{Z}$ is ergodic $[6,7]$. 


\section{Ergodic Measures: A NEW APPROACH}

Assume that there exists a holomorphic, in parameter $\omega \in \mathbb{C}_{+}$, function $\varrho_{\omega} \in$ $\mathrm{H}_{2}\left(\mathbb{C}_{+} ; \mathbb{C}\right)$, satisfying the following identity

$$
\hat{T}_{\varphi} \varrho_{\omega}=\varrho_{\tilde{\varphi}(\omega)}
$$

valid for any $\omega \in \mathbb{C}_{+}$for some induced transformation $\mathbb{C}_{+} \ni \omega \mapsto \tilde{\varphi}(\omega) \in \mathbb{C}_{+}$. If we now take $\omega:=\bar{\omega} \in \mathbb{C}_{+}$being a fixed point of the mapping $\tilde{\varphi}: \mathbb{C}_{+} \rightarrow \mathbb{C}_{+}$, then an easy conclusion from 3.1 gives rise to the condition $\hat{T}_{\varphi} \varrho_{\bar{\omega}}=\varrho_{\bar{\omega}}$, meaning that the expression

$$
d \mu(x):=\operatorname{Im} \varrho_{\bar{\omega}}(x) d x
$$

is a searched invariant measure for the transformation $\varphi: \mathbb{R} \rightarrow \mathbb{R}$. There exists still no general rule of constructing such functions $\varrho_{\omega} \in H_{2}\left(\mathbb{C}_{+} ; \mathbb{C}\right)$ analytic in $\omega \in \mathbb{C}_{+}$ and related induced mappings $\tilde{\varphi}: \mathbb{C}_{+} \rightarrow \mathbb{C}_{+}$. Nevertheless, for solving this problem one can adapt some natural motivations related to the exact functional form of the determining Frobenius-Perron operator $\hat{T}_{\varphi}: L_{2}(\mathbb{R} ; \mathbb{R}) \rightarrow L_{2}(\mathbb{R} ; \mathbb{R})$. To explain this, let us consider the following Boole type transformation:

$$
\mathbb{R} \ni \varphi(y):=\alpha y+a-\frac{\beta}{y-b} \in \mathbb{R},
$$

where $a, b \in \mathbb{R}$ and $\beta \in \mathbb{R}_{+}$. It is easy to observe that the Frobenius-Perron operator action on any $\varrho_{\omega} \in H_{2}\left(\mathbb{C}_{+} ; \mathbb{C}\right)$ can be represented as follows:

$$
\begin{aligned}
\hat{T}_{\varphi} \varrho_{\omega}:=\varrho_{\omega}\left(y_{+}\right) y_{+}^{\prime}+\varrho_{\omega}\left(y_{-}\right) y_{-}^{\prime} \\
=\frac{\left(\omega-y_{+}\right) \varrho_{\omega}\left(y_{+}\right)\left(\omega-y_{-}\right) y_{-}^{\prime}}{\left(\omega-y_{+}\right)\left(\omega-y_{-}\right)}+\frac{\varrho_{\omega}\left(y_{-}\right)\left(\omega-y_{+}\right)\left(\omega-y_{-}\right) y_{-}^{\prime}}{\left(\omega-y_{+}\right)\left(\omega-y_{-}\right)} \\
=\frac{k\left(\omega-y_{-}\right) y_{+}^{\prime}+k\left(\omega-y_{+}\right) y_{-}^{\prime}}{\left(\omega-y_{+}\right)\left(\omega-y_{-}\right)}=\frac{-k\left[\left(\omega-y_{+}\right)\left(\omega-y_{-}\right)\right]^{\prime}}{\left(\omega-y_{+}\right)\left(\omega-y_{-}\right)} \\
=-k \frac{d}{d x} \ln \left[\left(\omega-y_{+}\right)\left(\omega-y_{-}\right)\right],
\end{aligned}
$$

where we put, by definition,

$$
\varrho_{\omega}(x)=\frac{k}{\omega-x}
$$

for all $\omega \in \mathbb{C}_{+} \backslash\{x\}, x \in \mathbb{R}$, and some parameter $k \in \mathbb{R}$. As a result of (3.5) one can take

$$
\varrho_{\omega}\left(y_{+}\right)\left(\omega-y_{+}\right)=k=\varrho_{\omega}\left(y_{-}\right)\left(\omega-y_{-}\right)
$$

for all $x \in \mathbb{R}$ and $\omega \in \mathbb{C}_{+}$. Since the root functions $y_{+}, y_{-}: \mathbb{R} \rightarrow \mathbb{R}$ satisfy, by definition, the same equation

$$
\omega\left(y_{ \pm}(x)\right)=x,
$$


for all $x \in \mathbb{R}$, the following identity for all $\omega \in \mathbb{C}_{+}$is easily inferred from (3.7) owing to the general form of (3.3):

$$
\alpha\left(\omega-y_{+}\right)\left(\omega-y_{-}\right) \equiv[\varphi(\omega)-x](\omega-b),
$$

where

$$
y_{+}(x)+y_{-}(x)=b+\frac{x-a}{2}, \quad y_{+}(x) y_{-}(x)=\frac{b x-a b-\beta}{2} .
$$

Whence, taking into account the expression (3.4), one gets that

$$
\begin{aligned}
\hat{T}_{\varphi} \varrho_{\omega} & =-k \frac{d}{d x} \ln ([\varphi(\omega)-x](\omega-b)) \\
& =\frac{k(\omega-b)}{[\varphi(\omega)-x](\omega-b)}=\frac{k}{\varphi(\omega)-x}=\varrho_{\varphi(\omega)},
\end{aligned}
$$

for all $x \in \mathbb{R}$ and $\omega \in \mathbb{C}_{+}$. Therefore, the induced mapping $\tilde{\varphi}: \mathbb{C}_{+} \rightarrow \mathbb{C}_{+}$is exactly the transformation $\varphi: \mathbb{C}_{+} \rightarrow \mathbb{C}_{+}$, extended naturally from the axis $\mathbb{R}$ on the complex plane $\mathbb{C}_{+}$.

Let now $\tilde{\omega} \in \mathbb{C}_{+}$be a fixed point of the induced mapping $\varphi: \mathbb{C}_{+} \rightarrow \mathbb{C}_{+}$, that is $\varphi(\bar{\omega})=\bar{\omega} \in \mathbb{C}_{+}$. Then from (3.10) one finds that $\hat{T}_{\varphi} \varrho_{\bar{\omega}}=\varrho_{\bar{\omega}}$, or the corresponding invariant quasi-measure on $\mathbb{R}$ has the form

$$
d \mu(x):=\operatorname{Im} \frac{k d x}{\bar{\omega}-x}
$$

for all $x \in \mathbb{R}$ and some suitable parameter $k \in \mathbb{C}$. As $\operatorname{Im} \varrho_{\bar{\omega}} \in L_{2}\left(\mathbb{R} ; \mathbb{R}_{+}\right)$at any $\bar{\omega} \in \mathbb{C}_{+} \backslash \mathbb{R}$ and some $k \in \mathbb{C}$, then the invariant quasi-measure (3.11) becomes a true invariant measure. The obtained result can be formulated as the following theorem.

Theorem 3.1. The quasi-measure (3.11) is invariant with respect to transformation (3.3) for any $\alpha \in \mathbb{R}_{+} \backslash\{1\}$; for $\alpha=1$ at the condition $a \neq 0, \operatorname{Im} k \neq 0$, it is reduced upon the set $\mathbb{R} / \pi \mathbb{Z}$, being equivalent to the standard Gauss measure.

Proof. Indeed, the searched infinitesimal quasi-measure $d \mu(x)$ exist if there exists at least one fixed point of the equation $\varphi(\omega)=\omega$ for $\omega \in \mathbb{C}_{+}$. If $\alpha \neq 1$, this equation is equivalent to

$$
(\alpha-1) \omega^{2}-\omega[(\alpha-1) b-a]-(a b+\beta)=0,
$$

always possessing a solution $\bar{\omega} \in \mathbb{C}_{+}$, for which $\varphi(\bar{\omega})=\bar{\omega}$. At $\alpha=1$ the unique solution $\bar{\omega}=(a b+\beta) / a \in \mathbb{R}$ exists only at $a \neq 0, \operatorname{Im} k \neq 0$, at which the quasimeasure (3.11) becomes degenerate, thus reducing to the standard Gauss measure $[3,7]$ on $\mathbb{R} / \pi \mathbb{Z}$.

The Theorem 3.1 states only that quasi-measure (3.11) is invariant with respect to the transformation (3.3), thereby the ergodicity if any still needs to be proved separately, on which we plan to do in detail in another place. Below we will proceed to study the general case of transformation (1.1), searching for a suitable invariant quasi-measure becoming a measure at some $\bar{\omega} \in \mathbb{C}_{+} / \mathbb{R}, k \in \mathbb{C}$. 


\section{INVARIANT MEASURES: THE GENERAL CASE}

Consider the following equation

$$
\varphi(y)=x,
$$

where $x, y \in \mathbb{R}$ and the mapping $\varphi: \mathbb{C}_{+} \rightarrow \mathbb{C}_{+}$is given by expression (1.1) at some still fixed integer $N \in \mathbb{Z}_{+} \backslash\{1\}$. The equation (4.1) can be rewritten as

$$
\alpha \prod_{j=1}^{N+1}\left(y-y_{j}\right)=[\varphi(y)-x] \prod_{j=1}^{N}\left(y-b_{j}\right)
$$

for all $x, y \in \mathbb{R}$ and some functions $y_{j}: \mathbb{R} \rightarrow \mathbb{R}, j=\overline{1, N+1}$. Relation (4.2) is naturally extended to the complex plane $\mathbb{C}_{+}$as

$$
\alpha \prod_{j=1}^{N+1}\left(\omega-y_{j}\right)=[\varphi(\omega)-x] \prod_{j=1}^{N}\left(\omega-b_{j}\right)
$$

for any $\omega \in \mathbb{C}_{+}$.

Consider now relation (3.1), similarly to Section 3 :

$$
\begin{aligned}
& \hat{T}_{\varphi} \varrho_{\omega}= \sum_{j=1}^{N} \varphi(\omega)\left(y_{j}\right) y_{j}^{\prime}=\sum_{j=1}^{N+1} \frac{\varphi(\omega)\left(y_{j}\right)\left(\omega-y_{j} \prod_{k \neq j}^{N+1}\left(\omega-y_{k}\right) y_{j}^{\prime}\right.}{\prod_{k=1}^{N+1}\left(\omega-y_{k}\right)} \\
&=\sum_{j=1}^{N+1} \frac{\varphi(\omega)\left(y_{j}\right)\left(\omega-y_{j} \prod_{k \neq j}^{N+1}\left(\omega-y_{k}\right) y_{j}^{\prime}\right.}{\prod_{k=1}^{N+1}\left(\omega-y_{k}\right)}=\sum_{j=1}^{N+1} \frac{k \prod_{k \neq j}^{N+1}\left(\omega-y_{k}\right) y_{j}^{\prime}}{\prod_{k=1}^{N+1}\left(\omega-y_{k}\right)} \\
&=-k \frac{\frac{d}{d x} \prod_{k \neq j}^{N+1}\left(\omega-y_{k}\right)}{\prod_{k=1}^{N}\left(\omega-y_{k}\right)}=-k \frac{d}{d x} \ln \prod_{k=1}^{N+1}\left(\omega-y_{k}\right),
\end{aligned}
$$

where we have put, as before,

$$
\varrho_{\omega}\left(y_{j}\right)\left(\omega-y_{j}\right)=k,
$$

for all $j=\overline{1, N+1}, \omega \in \mathbb{C}_{+}$, and some parameters $k \in \mathbb{C}$. This evidently means that

$$
\varrho_{\omega}(y)=\frac{k}{\omega-y}
$$

for any $y \in \mathbb{R}$ and $\omega \in \mathbb{C}_{+}$.

Having substituted now expression (4.3) into (4.4), one gets easily that

$$
\hat{T}_{\varphi} \varrho_{\omega}(x)=\frac{k}{\varphi(\omega)-x}=\varrho_{\varphi(\omega)}(x),
$$

for all $x \in \mathbb{R}$ and any $\omega \in \mathbb{C}_{+}$. Thus, the invariant quasi-measure for the discrete dynamical system (1.1) will be given by the same expression (3.11), where $\bar{\omega} \in \mathbb{C}_{+}$ 
is some fixed point of the mapping $\varphi: \mathbb{C}_{+} \rightarrow \mathbb{C}_{+}$. This means that

$$
\alpha \bar{\omega}+a-\sum_{j=1}^{N} \frac{\beta_{j}}{\bar{\omega}-b_{j}}=\bar{\omega}
$$

or, equivalently,

$$
\alpha \bar{\omega} \prod_{j=1}^{N}\left(\bar{\omega}-b_{j}\right)+a \prod_{j=1}^{N}\left(\bar{\omega}-b_{j}\right)-\sum_{j=1}^{N} \beta_{j} \prod_{k \neq j}^{N}\left(\bar{\omega}-b_{k}\right)=\bar{\omega} \prod_{j=1}^{N}\left(\bar{\omega}-b_{j}\right),
$$

for some $\bar{\omega} \in \mathbb{C}_{+}$. Assume now that $\alpha \neq 1$; then it is easy to find that the algebraic equation (4.9) possesses exactly $N+1 \in \mathbb{Z}_{+}$roots, which can be used for constructing the invariant quasi-measure (3.11). The case $\alpha=1$ gives rise to the condition

$$
a \prod_{j=1}^{N}\left(\bar{\omega}-b_{j}\right)=\sum_{j=1}^{N} \beta_{j} \prod_{k \neq j}^{N}\left(\bar{\omega}-b_{k}\right),
$$

which always possesses roots if $N \geq 2$ and $a \in \mathbb{R}$ is arbitrary. Thereby, one can formulate the following theorem for the case $N \geq 2$.

Theorem 4.1. Expression (3.11) at some $k \in \mathbb{C}$ determines, in general, the infinitesimal invariant quasi-measure for the generalized Boole transformation (1.1) at all $N \geq 2$ with arbitrary parameters $a, b_{j} \in \mathbb{R}$ and $\alpha, \beta_{j} \in \mathbb{R}_{+}, j=\overline{1, N+1}$.

It is an important task now to separate from the obtained set of invariant quasimeasures (3.11) those which are positively defined and ergodic with respect to transformation (1.1) at $N \geq 2$. The positivity condition simply means that the determining equation (4.9) must possess at least one pair of complex conjugated roots with a nontrivial imaginary part. Having analyzed, concerning this criteria, roots of equation (4.9) one can find that the following statement analogous to that proved in [6] holds.

Theorem 4.2. The generalized Boole transformation (1.1) for any $N \geq 1$ is necessarily ergodic with respect to the measure (3.11) at some $\bar{\omega} \in \mathbb{C}_{+} \backslash \mathbb{R}$ and $k \in \mathbb{C}$ iff $\alpha=1$ and $a=0$. If $\alpha=1$ and $a \neq 0$, the transformation (1.1) is not ergodic being totally dissipative, that is the wandering set $\mathscr{D}(\varphi):=\bigcup \mathscr{W}(\varphi)=\mathbb{R}$, where $\mathscr{W}(\varphi) \subset \mathbb{R}$ are subsets such that all sets $\varphi^{-n}(\mathscr{W}), n \in \mathbb{Z}$, are disjoint.

SKeTch OF PROOF. It is easy to see that for $N \geq 2$ at $\alpha=1$ and $a=0$ the determining algebraic equation (4.9) always possesses exactly $N-1 \in \mathbb{Z}_{+}$real roots $\bar{\omega}_{j} \in \mathbb{R}$, $j=\overline{1, N-1}$. Therefore, the invariant quasi-measure expression (3.11) is degenerate for $\bar{\omega}_{j} \in \mathbb{R}, j=\overline{1, N-1}$, giving rise to the conclusion that the corresponding invariant measure $d \mu(x)=d x, x \in \mathbb{R}$, is the standard Lebesgue measure on $\mathbb{R}$. Its ergodicity with respect to transformation (1.1) then follows from the fact that the corresponding dissipative set $\mathscr{D}(\varphi)=\varnothing$ and the unique invariant set subalgebra $I(\varphi)=\mathbb{R}$. 
The statements similar to above can be formulated for the most generalized Boole type transformation

$$
\mathbb{R} \ni y \rightarrow \varphi(y):=\alpha y+a+\int_{\mathbb{R}} \frac{d v(s)}{s-y} \in \mathbb{R},
$$

where $a \in \mathbb{R}, \alpha \in \mathbb{R}_{+}$and a measure $v$ on $\mathbb{R}$ has the compact support $\operatorname{supp} v \subset \mathbb{R}$, being such that the following natural conditions [3]

$$
\int_{\mathbb{R}} \frac{d v(s)}{1+s^{2}}=a, \int_{\mathbb{R}} d v(s)<\infty,
$$

hold. Concerning the extension of the transformation (4.11) on the upper part $\mathbb{C}_{+}$of the complex plane $\mathbb{C}$ in such a way that $\operatorname{Im} \varphi(\omega) \geq 0$ for all $\omega \in \mathbb{C}_{+}$, the following representation

$$
\varphi(\omega)=\alpha \omega+a+\int_{\mathbb{R}} \frac{1+s \omega}{s-\omega} d \sigma(s)
$$

holds [3,11], where a measure $d \sigma$ on $\mathbb{R}$ is closely related to the measure $d v$.

The general properties of mapping (4.13) were in part studied in [6] in the framework of the theory of inner functions. The invariant measures corresponding to (4.11) and their ergodic properties can be also treated effectively making use of the analytical and spectral properties of the associated Frobenius-Perron transfer operator (1.3). These aspects of the problem being are as important as interesting, and are planned to be studied elsewhere.

\section{Acknowledgements}

The authors are cordially grateful to professors D. Blackmore (NJ, USA), F. Pszytycki (Warsów) and T. Downarowicz (Wroclaw) for valuable discussions of the ergodic measure properties related to the generalized Boole transformations.

\section{REFERENCES}

[1] Boole, G. On the comparison of transcendents with certain applications to the theory of definite integrals, Philos Trans. Royal Soc. London, 147 (1957), 745-803.

[2] Adler, R. AND Weiss, B.: The ergodic, infinite measure preserving transformation of Bool, Israel Journal of Math., 16 (1973), 263-278.

[3] Aaronson, J.: Ergodic theory for inner functions of the upper half plane, Ann. Inst. H. Poincaré, BXIV (1978), 233-253.

[4] Aaronson J.: A remark on this existence of inner functions, J. London Math. Soc, 23 (1981), $469-474$.

[5] Aaronson, J.: The eigenvalues of nonsingular transformations, Israel J. Math., 45 (1983), 297312.

[6] Aaronson, J.: An Introduction to Infinite Ergodic Theory, Amer. Math. Soc, 1997.

[7] Каток, A. and Hasselblatt, B.: Introduction to the Modern Theory of Dynamical Systems, Cambridge University Press, Cambridge, 1997.

[8] Prykarpatsky A. K.: On invariant measure structure of a class of ergodic discrete dynamical systems, Nonlinear Oscillations, 3 (2000), No. 1, 78-83. 
[9] Prykarpatsky, A. K. and Brzychczy, S.: On invariant measure structure of a class of ergodic discrete dynamical systems, Proceedings of the International Conference SCAN 2000/Interval 2000, September 19-22, Karlsruhe, Germany.

[10] Pollycott, M. and Yari, M.: Dynamical Systems and Ergodic Theory, London Math. Society, Cambridge University Press, 1998.

[11] Ablowitz, M. J. and Fokas, A. S.: Complex Variables: Introduction and Applications, Cambridge University Press, 1997.

\section{Authors' addresses}

\section{Anatoliy K. Prykarpatsky:}

The AGH University of Science and Technology, Department of Applied Mathematics, Krakow 30059, Poland

\section{Jacek Feldman:}

The AGH University of Science and Technology, Department of Applied Mathematics, Krakow 30059, Poland 